\title{
3-D Microscale Geometry of Apple Tissue using X-ray Computed Microtomography
}

\author{
Mendoza, F. ${ }^{1}$, Verboven, P. $^{1}$, Ho, Q. T. ${ }^{1}$, Mebatsion, H. K. ${ }^{1}$, Nguyen, T. A., Wevers, M. ${ }^{2}$, \\ Nicolaï, B. ${ }^{1}$
}

\begin{abstract}
${ }^{1}$ BIOSYST - MeBioS, Faculty of Bioscience Engineering, Katholieke Universiteit Leuven, W. de Croylaan 42, BE-3001, Leuven, Belgium. Phone: +32 16322384 - Fax: +32 16 322955. E-mail: Fernando.Mendoza@biw.kuleuven.be.

${ }^{2}$ Mechanical Metallurgy Section, Department of Metallurgy and Materials Engineering (MTM), Katholieke Universiteit Leuven, Kastelpark Arenberg 44, BE-3001, Leuven, Belgium. Phone: +32 16 321303 - Fax: +32 16321990.
\end{abstract}

\begin{abstract}
The microstructure and the connectivity of the pore space are important variables for better understanding of the complex transport phenomena that occur in apple tissue. In this study, we present geometrical properties (i.e., porosity and specific surface area) and spatial distributions for nodal pore volume, nodal pore size, tortuosity, coordination number, and pore path length obtained from a representative elementary volume (REV) of $1.3 \mathrm{~mm}^{3}$ of apple tissue. For comparison, two different regions of the sample were analyzed: in the fleshy part of the apple ('cortex tissue', $\sim 6 \mathrm{~mm}$ from the peel) and near to the core line ('core tissue', $\sim 10 \mathrm{~mm}$ from the core center). The apple tissue was imaged using X-Ray computed microtomography with a pixel size of $8.5 \mu \mathrm{m}$. The spatial distributions were computed based upon three dimensional medial axis analysis of the void space in the image and using the average of six apples (variety 'Jonagold').

Results showed that microtomography provides micrometer resolution to quantitatively analyze and characterize apple tissue. The minimum REV of apple tissue was estimated to be $1.3 \mathrm{~mm}^{3}$. Comparisons among the parameters extracted from the two analyzed regions showed significant differences $(\mathrm{p}<0.05)$. The porosity and specific surface area for cortex tissue and core tissue were $31 \pm 3 \%$ and $1.8 \times 10^{-2} \pm 1.2 \times 10^{-3} \mu \mathrm{m}^{-1}$ and $17 \pm 4 \%$ and $1.4 \times 10^{-2}$ $\pm 2.7 \times 10^{-3} \mu \mathrm{m}^{-1}$, respectively. The complexity and variability of the pore space network tissue taken from two different regions of the apple, highlighted the difficult of creating a unique network for 3-D representation of apple tissue, and therefore, the physically-realistic pore network characterization of apple tissue cannot be adequately represented using simple average parameters of the total tissue.
\end{abstract}

Keywords. Pore scale characterization, X-ray computerized microtomography, imaging techniques, elementary volume, apple tissue. 


\section{Introduction}

The geometry of the structure of the pore space plays a fundamental role in governing fluid and gas transport through porous media of any cellular tissue (Celia et al., 1995; Kuroki et al., 2004). However, establishing the relationships between the geometrical properties of the pore structure of cellular materials such as agricultural products and foods, and the bulk flow properties of the medium have been difficult for two main reasons: the difficulty in making direct measurements on the pore space, and the random nature of its structure (Khan \& Vincent 1990; Thovert et al., 1993; Maire et al., 2003).

$\mathrm{X}$-ray microtomography is relatively a new technique which has found potential applications in food science research and quality evaluation. The technique allows visualization and analysis of the architecture of cellular materials with an axial and lateral resolution down to a few micrometers, and without sample preparation or chemical fixation (van Dalen et al., 2003). X-rays are short wave radiations, which can penetrate through fruit. The level of transmissions of these rays depends mainly on the mass density and mass absorption coefficient of the material (Salvo et al., 2003). It is well known that the density of many fruits increases with maturity (Baoping, 1999).

Using X-Ray tomography, Barcelon et al. (1999) detected internal quality changes in peaches, and Lammertyn et al. (2003) investigated core breakdown disorders in 'Conference' pears based on their mass density variations during storage. Kuroki et al. (2004) obtained 3-D spatial information about gas filled intercellular spaces in sarcocarp of cucumber fruit, and also investigated the structural changes of these spaces during storage. Recently, Babin et al. (2005) studied the microstructure of cellular cereal products captured by synchrotron radiation and the relationships with their mechanical properties based on finite element simulations.

The motivation of this study was found in the necessity to extract realistic and statistical 3-D internal data of apple tissue at micron resolution. The extracted 3-D data from radiographic images can be analyzed using the free package software 3DMA-Rock developed by Lindquist (1999). These algorithms have been successfully tested in many investigations using different model materials, and they have been mainly used in the microstructure studies of variety of sandstones, cores and basalt rocks.

The objectives of this study were: (1) to evaluate the representative elementary volume (REV) of apple tissue over which statistical average parameters of its microstructure can be extracted using X-Ray computed microtomography at 8.5 micron resolution, and (2) to quantitatively characterize and compare the pore-network structure of apple tissue (variety 'Jonagold') taken from two different regions of the fruit: near to the peel (cortex tissue, $\sim 7 \mathrm{~mm}$ from the peel) and near to the line core (core tissue, $\sim 10 \mathrm{~mm}$ from the core center). We present geometrical properties (i.e., porosity and specific surface area) and spatial distributions of the nodal pore volume, effective pore radio, tortuosity, coordination number, and pore path length obtained for a $1.3 \mathrm{~mm}^{3}$ volume of apple tissue.

\section{Materials and Methods}

\section{Apple samples}

Six apples (variety 'Jonagold'), $7.5 \mathrm{~cm}$ diameter, stored under optimal conditions $\left(0.8^{\circ} \mathrm{C}\right.$, $2.5 \% \mathrm{CO}_{2}$, and $1 \% \mathrm{O}_{2}$ ) were selected from a commercial orchard in Belgium. For analysis, samples were taken in duplicate from each apple $\left(0^{\circ}\right.$ and $180^{\circ}$ around of the fruit $)$ at two 
different positions: in the flesh of the apple (cortex tissue, $\sim 7 \mathrm{~mm}$ from the peel) and near to the core line (between cortex and core tissue, $\sim 10 \mathrm{~mm}$ from the core center). Each sample was cut into cylinder with $5 \mathrm{~mm}$ diameter and closed in a plastic tube to avoid dehydration without any special preparation.

\section{Data collection}

The samples were scanned using a high resolution desk-top X-ray micro-CT system, SkyScan 1072 , with a linear resolution of $8.5 \mu \mathrm{m}$ by pixel, operating at a voltage of $63 \mathrm{kV}$, current of $156 \mu \mathrm{A}$, an exposure time of $8.4 \mathrm{sec}$, and scanned over the interval $0^{\circ}-180^{\circ}$ using a $0.9^{\circ} \mathrm{scan}$ step. These experimental conditions were the same for all the analyzed samples since they were optimized to enhance both the contrast and resolution of the images. The digitalized Xray shadow projections of the 3 -D object with $1024 \times 1024$ pixels size (12 bit) were processed to obtain reconstructed cross-section images with a 256 gradations (8 bit) using a mathematical algorithm based on the back-projection procedure. Thus, a complete stack of 2D cross-sections ( 200 slices) of the object with an $8.5 \mu \mathrm{m}$ interslice distance is obtained and can be used for reconstruction and analysis of the full 3-D porous media of apple tissue.

\section{Image processing}

From each stack of images, a representative elementary volume was subjected to image analysis. The reconstructed images were pre-processed using the Image Processing Toolbox of MATLAB 7.0 (The MathWorks, Inc., USA) to render the apple tissue microstructure as 2$\mathrm{D}$ stacks and 3-D images. Thus, 3-D image consisted of 128 points or small voxels (volume elements) with a length voxel side of $8.5 \mu \mathrm{m}$. Quantitative analysis was carried out by using the free software package 3DMA - Rock Primer ver. 08/05 (Lindquist, 1999). The procedure mainly consisted of the two following steps and algorithms:

a) Image segmentation. Representation of the geometry of the void space requires a voxel-byvoxel determination of the phase type (image segmentation). Here, the thresholding method developed by Oh \& Lindquist (1999) was used to segment the apple tissue images into pores and cellular material. The algorithm utilizes the spatial covariance of the image in conjunction with indicator kriging to determine object edges. Use of indicator kriging makes the thresholding local (based on two threshold values, $T_{0}$ and $T_{1}$ ) and guarantees smoothness in the threshold surface. The segmentation process is done fully in three dimensions, and not slice-wise. For the thresholding step of apple tissue images, the gray threshold values were set at 40 and 70. According to this thresholding window, a partial identification of the image was done as: pores being voxels having gray values not greater than 40 , and voxels of gray value greater than 70 being judged as cellular material. Finally, the remainder voxels of the population were identified by indicator kriging.

b) Image skeletonization. In order to extract geometrical statistics from the 3-D apple tissue networks, the reconstructed segmented images were first simplified to their medial axes or 'skeletons'. The medial-axis offers a skeletal representation of the pore surface, while preserving its original topology and geometry, so that the network geometry can be efficiently analyzed. The pore-network analysis algorithms are based on the three dimensional skeletonization of the pore space in the form of nodes connected to paths. Details of the skeletonization algorithm used in this work can be found in Lee et al. (1994), Lindquist et al. (1996), and Lindquist (1999). Only a brief overview of the technique, and relevant terminology, will be presented here. 
For a digitalized pore network in 3-D space, the medial axis consist of a network of tortuous 'paths' and 'clusters'. A 'path' is defined as a connected string of filled voxels (3-D pixels), each voxel having exactly two filled neighbors. A 'cluster' is defined as a set of medial axis voxels, each with at least three neighbors lying in the same cluster. Thus, a vertex on the medial axis, where several pore paths meet, corresponds to the location of a 'nodal pore'. The number of paths meeting at a cluster (nodal pore) is termed the 'coordination number'.

Special precautions were taken to optimize the statistical reliability of the medial axis data. As it is defined relative to the object's surface, construction of the medial axis is sensitive to surface noise. Surface noise occurs in two forms, irregularities in an otherwise smooth digitized void-cellular surface, and disconnected clusters of void or cellular voxels which occur due to voxel misidentification. Irregularities in the void-cellular surface can have the same effect as dead-end pores, producing extraneous branches on the void medial axis. These extraneous branches must be identified and removed before the medial axis can be used to characterize the object. We employ a user set cutoff on the length of such branches to distinguish between true dead end pore paths and those produced by surface irregularities. If only that portion of the void space which supports fluid flow is to be investigate, all dead-end branches can be trimmed from the void space axis using the cluster-merge algorithm.

Isolated clusters of void phase voxels will appear as disconnected 'objects' each with their own skeleton piece. Since isolated regions of void phase may indeed be physical, it is much more difficult to distinguish between small isolated void space clusters which are physical and those which are artifacts; all isolated void clusters less than $1 \%$ volume size limit were re-identified as cellular phase. On the other side, isolated clusters of cellular voxels appear as 'cavities' within the void space. Similarly, all small isolated clusters less than $1 \%$ volume size were removed, re-identifying their voxels as void voxels. This produces error in the medial axis at the edges of the imaged volume in proportion to the ratio of boundary area to volume of the imaged region, but has the advantage of producing a medial axis for the void space that is only a union of one-dimensional curves (Lindquist, 1999).

\section{Representative elementary volume (REV)}

Considering that the computation time for 3-D images is dependent on the size of the analyzed image, it is frequently desirable for geometrical medial axis analysis to use a subregion of this original stack of radiographic images (in a form of a cylinder or a box). This sub-volume should be small enough so that it can be treated as a mathematical point at the continuum, and large enough to provide representation of the macroscopic properties of the porous media. The REV is defined as the volume over which a statistical average can be performed.

In order to ensure that the sample of concern is homogeneous and therefore representative, the porosity of 6 volume sizes $\left(2.6 \times 10^{-3}, 2 \times 10^{-2}, 0.16,1.3,10.4\right.$, and $\left.20.6 \mathrm{~mm}^{3}\right)$ taken from the cortex tissue of the apple was evaluated and analyzed in triplicate. The volumes for each repetition were obtained from the same stack of images dividing the entire 3-D image in a number of sub-volumes for each volume size. The procedure was implemented in Matlab and it works increasing the side length of the voxel in all directions until reach length of 16,32 , $64,128,256$, and 320 pixels (with $8.5 \mu \mathrm{m} / \mathrm{pixel}$ ) per side. Then the average porosity and standard deviations for this number of sub-volumes were calculated. 


\section{Extracted microstructural parameters}

From this previously determined REV, averages of the geometrical parameters and probability density distributions for the two analyzed regions of apple tissue were computed in duplicate from 6 samples. Porosity represents the void space as a fraction of the total volume of the sample, and it is calculated from binary segmented images by simple count of the voxels of the pore space divided by the total number of voxels. Specific surface area represents a measure of the interstitial surface area of the void-solid phase per bulk volume, and it is calculated by summing the areas of all pore-cellular material voxel faces, and dividing this area by the total image volume. Nodal pore volume represents the volume of the inscribed sphere inside of the nodal pore. Effective nodal pore radius represents the radio of the inscribed sphere inside of the nodal pore and can be calculated from the volume measurements using $V=(4 / 3) \pi R^{3}$. Tortuosity $(\tau)$ describes the void connectivity between two parallel faces of the sample, and it is calculated as the shortest paths along the medial axis for $x$-, $y$-, and $z$ - directions using $\tau \equiv l / \Delta$, where $l$ is the actual path length and $\Delta$ is the linear separation of the two parallel planes. Coordination number represents the number of paths (i.e. throats) connected to each pore body. Finally, pore channel length is a measure of the distance between the centers of any two adjacent (i.e. connected) nodal pores. The distance measured is not the straight line distance, but the distance along the middle (i.e. along the medial axis) of the pore channel connecting them.

\section{Statistical analysis}

Statistical evaluations for distribution fitting and differences between the extracted parameters (as average or spatial distributions) from cortex and core tissue were performed by means of MATLAB 7.0 (The MathWorks, Inc., USA). Applied methods were: the t-test (assuming unequal variances) and the no-parametric Kolmogorov-Smirnov test with 95\% confidence level. The evaluated distributions were:

a) Normal distribution:

$$
y=f(x \mid \mu, \sigma)=\frac{1}{\sigma \sqrt{2 \pi}} e^{\left(\frac{-(x-\mu)^{2}}{2 \sigma^{2}}\right)}
$$

where parameters $\mu, \sigma$, and $\sigma^{2}$ are the sample average, standard deviation and variance, respectively.

b) Lognormal distribution:

$$
y=f(x \mid \mu, \sigma)=\frac{1}{x \sigma \sqrt{2 \pi}} e^{\left(\frac{-(\ln x-\mu)^{2}}{2 \sigma^{2}}\right)}
$$

c) Gamma distribution:

The gamma distribution has the following relationship with the incomplete Gamma function:

$$
\Gamma(x \mid a, b)=\operatorname{gammainc}\left(\frac{x}{b}, a\right)
$$

For $b=1$ the gamma distribution and incomplete gamma function are identical. When $a$ is large, the gamma distribution closely approximates a normal distribution with the advantage 
that the gamma distribution has density only for positive real numbers. The definition of the gamma probability distribution function is:

$y=f(x \mid a, b)=\frac{1}{b^{a} \Gamma(a)} x^{(a-1)} e^{\frac{x}{b}}$

Where $\Gamma(\cdot)$ is the gamma function.

\section{Results \& discussion}

\section{Representative elementary volume analysis (REV)}

Figure 1 shows this result using the average porosity of three stacks of images of 'Jonagold' apples. All the analyzed sub-volumes show similar average porosities, however, the computed variability shows to be affected strongly by the size of the image. As will be expected this variability decreases when the size of the volume is increased. For the smallest volume $\left(2.6 \times 10^{-3} \mathrm{~mm}^{3}\right.$, equivalent to 16 pixels/side) the variability was $19.3 \%$ and for a volume of $10.4 \mathrm{~mm}^{3}$ (equivalent to $256 \mathrm{pixels} / \mathrm{side}$ ) was only $1.5 \%$. However, it can be observed that for the largest volume $\left(20.4 \mathrm{~mm}^{3}\right.$, equivalent to 320 pixels/side) the porosity slightly decreases and the variability increases. This will be explained considering that these stacks of images take in account regions closer to the borders of the sample which could be frequently damaged during the extraction, cutting and packing of the sample.

Therefore, from this analysis the minimum REV of apple tissue should be suggested to be 1.3 $\mathrm{mm}^{3}$ (equivalent to 128 pixels/side) which shows a reasonable variability of $2.7 \%$ using 3 apple samples. This image size was used for image processing and geometrical computations in the next steps of this investigation.

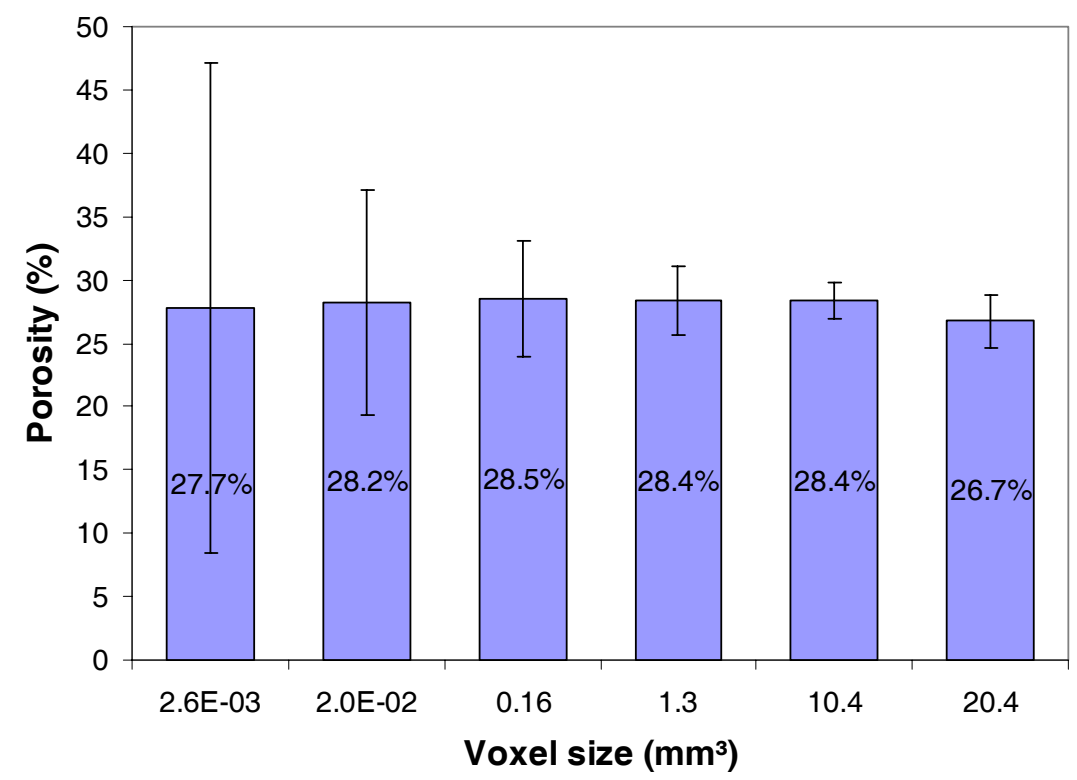

Figure 1. Representative elementary volume analysis (REV) using three stacks of images from the cortex of 'Jonagold' apples. 


\section{Graphic representation of apple tissue in the 'cortex' and the 'core' regions}

Figure 2 shows representative cross-sectional microtomography images from cortex tissue and core tissue taken from 6 different apples. As can be inferred from these figures, the investigated regions are characterized by different porous structures. In the cortex of the apple, the pores appear large and do not show much orientation. However, inwards, the pores appear elongated and showing some orientation. Also, heterogeneity in the porosity distribution of the bulk apple tissue samples is visually evident. The segmented images using these 12 apple samples with a REV cube of $1.3 \mathrm{~mm}^{3}$ had reported average porosity for cortex tissue and core tissue of $31 \pm 3 \%$ and $17 \pm 4 \%$, and average specific surface area of $1.8 \times 10^{-}$ ${ }^{2} \pm 1.2 \times 10^{-3} \mu \mathrm{m}^{-1}$ and $1.4 \times 10^{-2} \pm 2.7 \times 10^{-3} \mu^{-1}$, respectively. The two geometrical parameters of both tissues were significantly different $(\mathrm{P}$-value $<0.05)$.

\section{a) Cortex tissue}

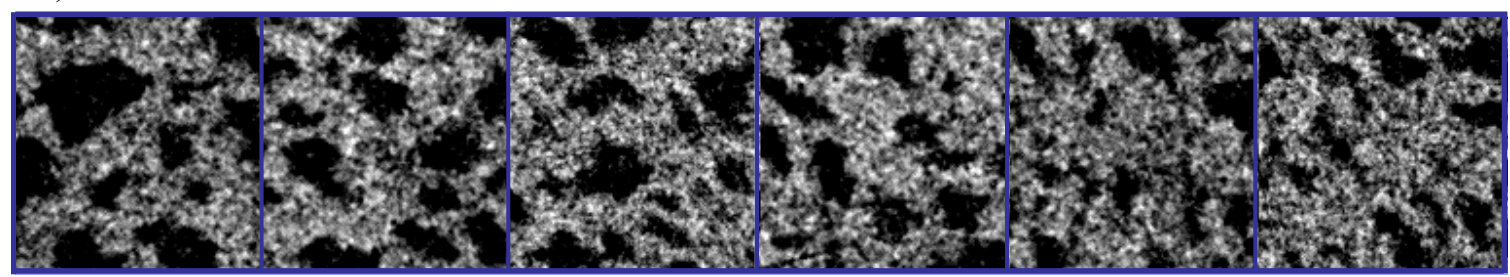

b) Core tissue

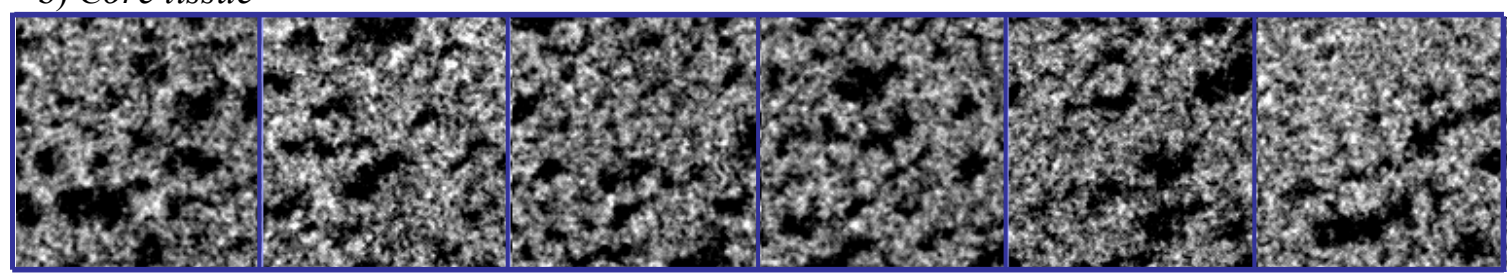

Figure 2. Representative 2-D raw microtomography images from (a) cortex tissue and (b) core tissue taken from 6 different apples of variety 'Jonagold' (black regions represent pores and gray regions represent cellular material).

Figure 3 shows representative 3-D images with a volume of $1.3 \mathrm{~mm}^{3}$ to illustrate the overall process of creating the segmented cube, percolating backbone, cellular microstructure surface, and tortuosity of shortest paths of apple tissue taken from the cortex. As discussed above, the percolating network has been corrected for noise by re-identifying the phase type of all isolated cellular and void voxel clusters to avoid spurious elements or non-percolating components of the pore space. The trimmed medial axis for the void space of this image is shown in Fig. $2 b$. 

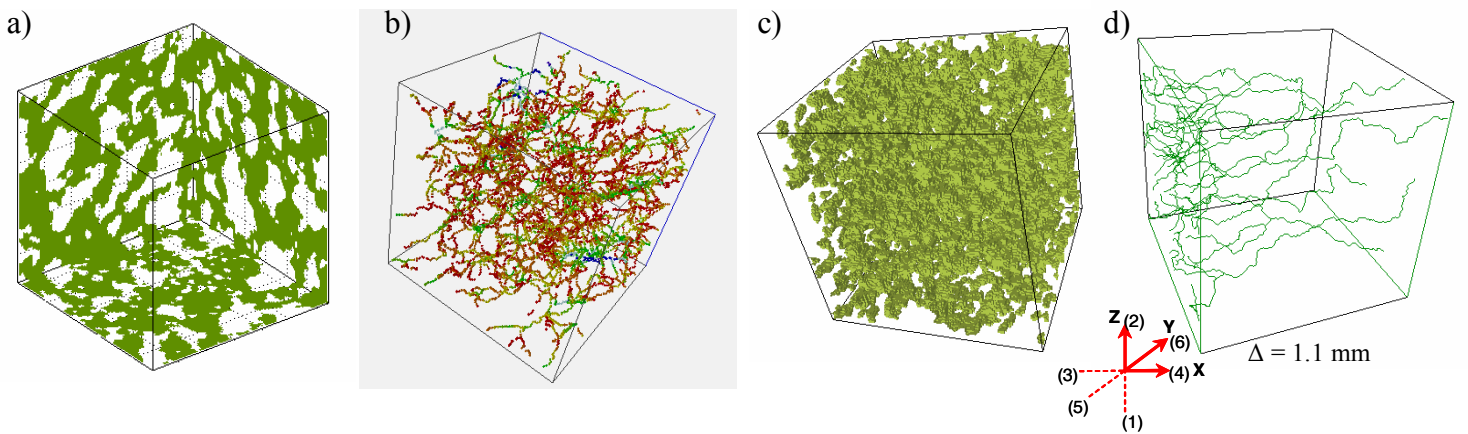

Figure 3. 3-D representation of the architecture of apple tissue taken from the cortex: a) 3-D binary cube from segmented images using the kriging-based algorithm (white regions represent pores and dark regions represent cellular material), b) 3-D percolating backbone or trimmed medial axis of pore space, c) 3-D pore-cellular material surface, obtained by the Marching Cubes algorithm (Lorense \& Cline, 1987), d) Geometrical tortuosity of shortest paths, in $x$-direction, from face (3) to face (4).

\section{Quantitative characterization of the cortex and core regions in apple tissue}

Figure 4 shows the distributions of nodal pore volumes for cortex and core tissue. The $x$-axis is plotted in log-scale since the original distribution is highly skewed, with a long tail to large volumes. Using a $\log$ transformation, the pore volumes cover approximately 5 orders of magnitude for both analyzed regions. Thus, Figure 4 suggests that a reasonable fitting model for both volume distributions is a log-normal distribution ( $\mathrm{P}$-value $>0.05$ ), with a mean value of the $\log$ (volume) of $-3.07=\log \left(0.00084 \mathrm{~mm}^{3}\right)$ and standard deviation of 0.91 for cortex tissue, and mean value of the $\log ($ volume $)$ of $-3.40=\log \left(0.0004 \mathrm{~mm}^{3}\right)$ and standard deviation of 0.81 for core tissue. Both distributions were significantly different $(\mathrm{P}-\mathrm{value}<0.05)$.

a)

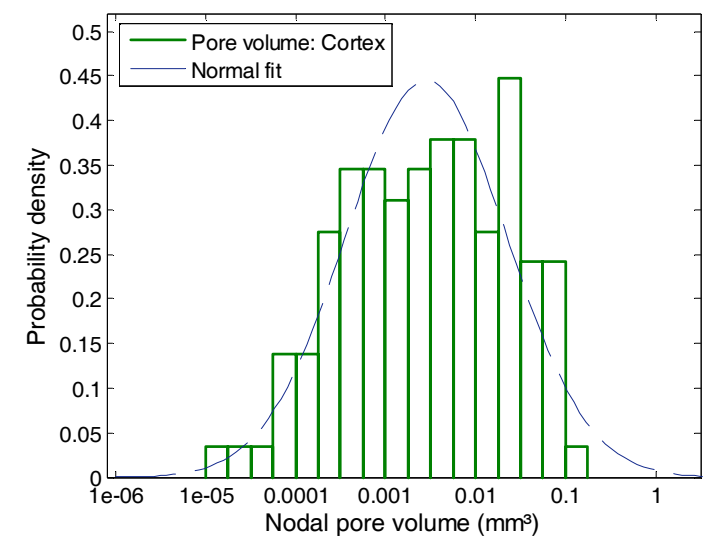

b)

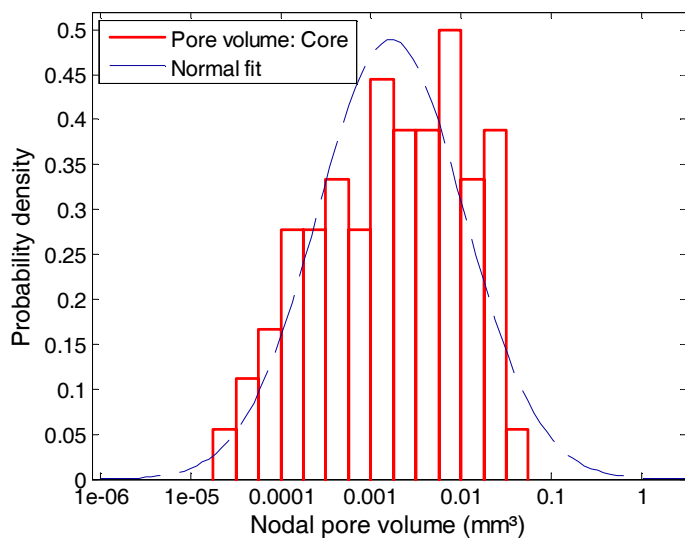

Figure 4. Measured distributions (each averaged over six $1.3 \mathrm{~mm}^{3}$ apple samples) of nodal pore volumes taken from: a) cortex tissue and b) core tissue. 
In Figure 5, we present the same distributions in terms of the effective nodal pore radius, assuming the spherical nodal pores. The average effective radius is $84.1 \pm 46.1$ and $68.1 \pm 30.9$ $\mu \mathrm{m}$ for cortex and core tissue, respectively. The large standard deviation reflects the skew in the distribution. In this case, the distributions for both analyzed regions are well described by a gamma fit (P-value $>0.05)$, and the two distributions were significantly different (Pvalue $<0.05)$.

a)

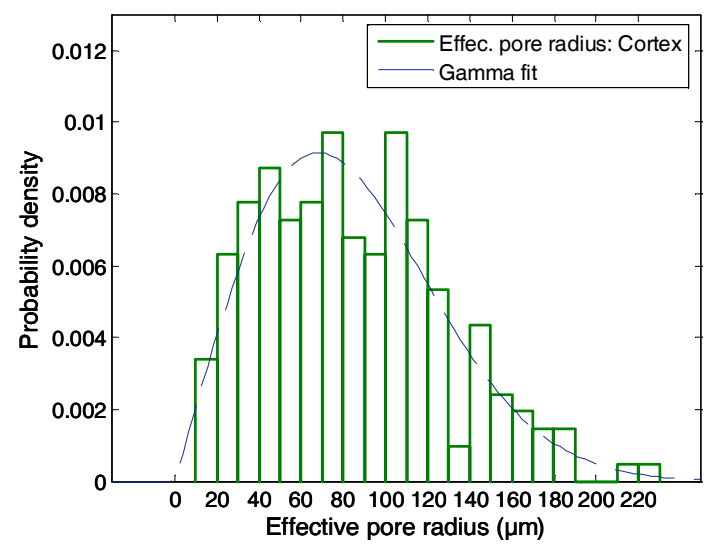

b)

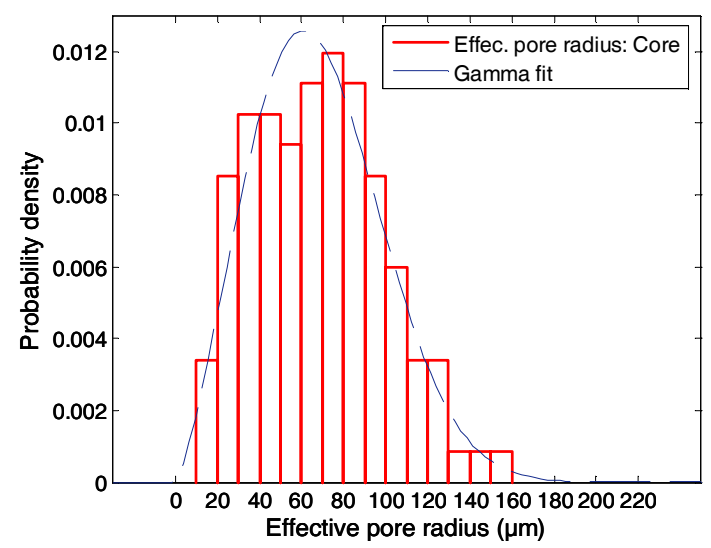

Figure 5. Measured distributions (each averaged over six $1.3 \mathrm{~mm}^{3}$ apple samples) of effective nodal pore radius assuming the spherical nodal pores taken from: a) cortex tissue and b) core tissue.

In Figure 6, we plot the tortuosity distributions of shortest paths along the medial axis linking the three parallel faces of a volume of $1.3 \mathrm{~mm}^{3}$. In this case, the average tortuosity estimation is $2.14 \pm 0.41$ and $1.94 \pm 0.30$ for cortex and core tissue, respectively. For both analyzed regions, the shape of the tortuosity probability density distribution is very well fitted by both lognormal and gamma distributions, which are shown superimposed over each distribution. Both tortuosity distributions were also shown to be significantly different ( $\mathrm{P}$-value $<0.05$ ).

a)

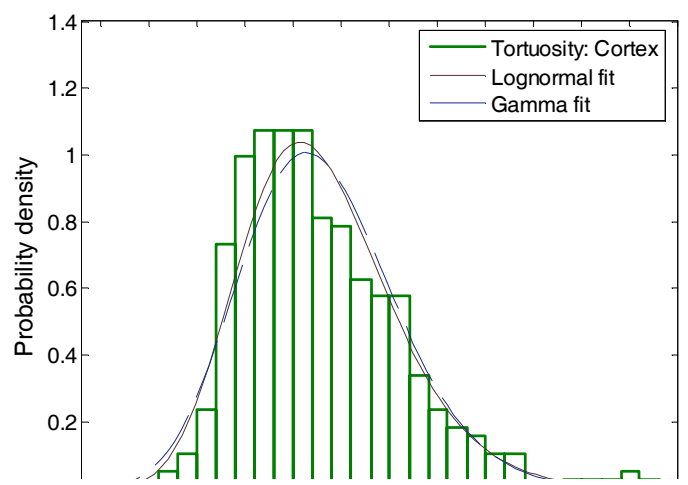

b)

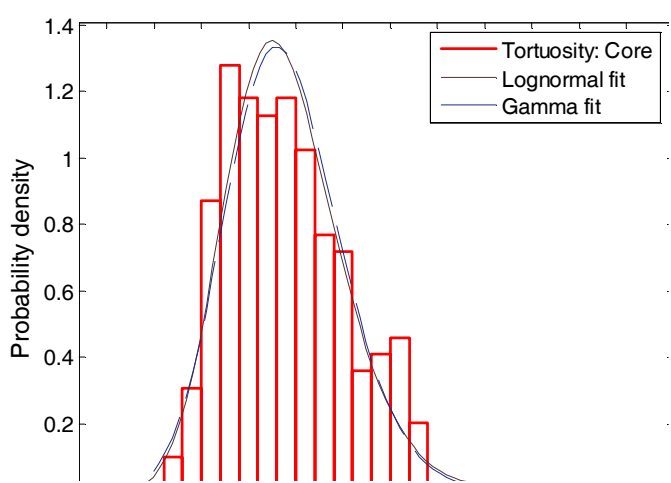

Figure 6. Tortuosity distribution for all shortest paths along the medial axis liking the three parallel faces $\left(x-, y-\right.$, and $z$-directions) of a $1.3 \mathrm{~mm}^{3}$. 
Of fundamental interest is the connectivity of the void paths. The connectivity has a critical influence on transport properties such as permeability, and its quantification is important for understanding microstructure-transport property relationships. Figure $7 \mathrm{a}$ shows the coordination number distribution measured from the medial axis as a form of describing the number of paths connected to each pore body. Over the range of coordination numbers from 1 to 10 , similar distribution trends are observed for both cortex and core tissue. Coordination number 3 branching predominates, occurring 2.9 times for cortex tissue and 2.7 for core tissue more frequently than coordination number 4 . Topologically a coordination number $3-$ branch can be viewed as one pore path dividing into two; while a coordination number 4branch can be viewed as two pore paths crossing. The average coordination number for the distribution of cortex tissue was slightly higher than for core tissue being their average values $4 \pm 2$ and $3 \pm 2$, respectively.

Figure $7 \mathrm{~b}$ shows the path length distributions of pores for each region. We see that both distributions are broad and skewed to large lengths, with similar trends over the range from 1 to $\sim 1100 \mu \mathrm{m}$. However, the distribution peaks for cortex and core tissue occur at path length of around 85 and $60 \mu \mathrm{m}$; and the average path lengths are 159.4 \pm 44.9 and 104.2 $\pm 43.4 \mu \mathrm{m}$, respectively. In network models, the pore channel length is commonly assumed to be constant. Here, significant differences $(\mathrm{P}$-value $<0.05)$ were found between channel length distributions of tissue near to the peel and tissue near to the core.

a)

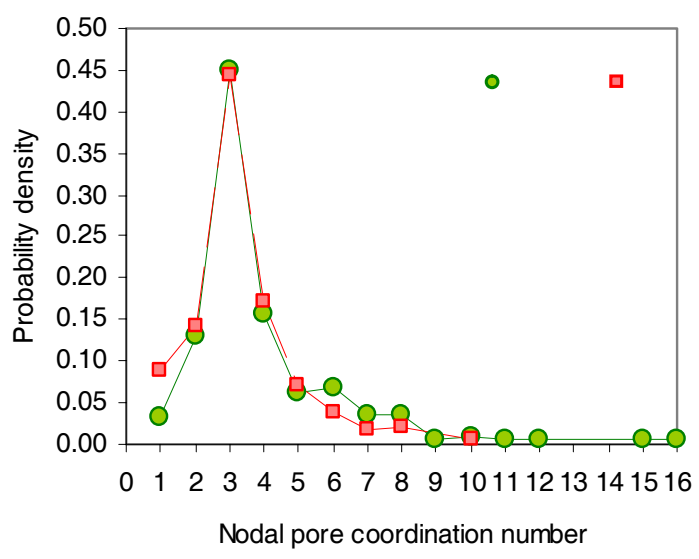

b)

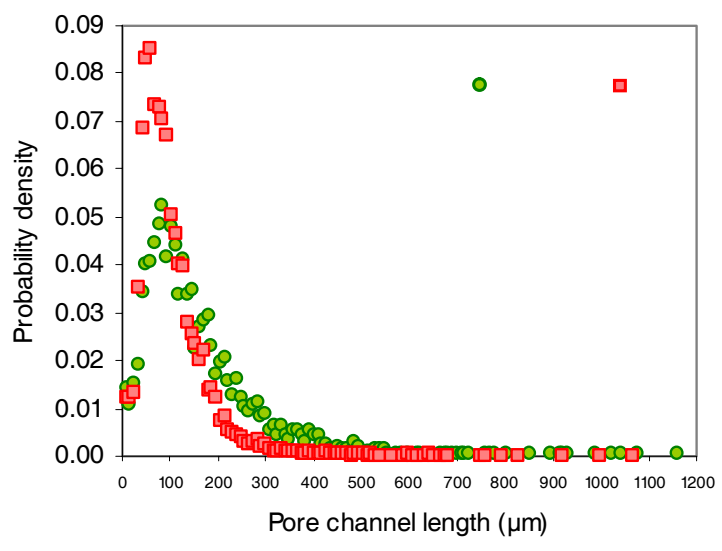

Figure 7. Distributions of pore network for: a) coordination numbers, b) and pathway lengths determined from the medial axis. Both distributions are broad and skewed to high coordination numbers and large lengths respectively, with similar distribution trends for cortex and core tissue. Coordination number 3 branching predominates, occurring 2.9 times for cortex tissue and 2.7 for core tissue more frequently than coordination number 4 . The distribution peaks for cortex and core tissue occur at path length of around 85 and 60 microns, respectively.

Another simpler measure of connectivity, explored here, is the number of disconnected medial axes found in the analyzed regions. This measure corresponds to the number of disconnected void spaces. Thus, the segmentation algorithm applied to these images using a $1.3 \mathrm{~mm}^{3}$ volume of apple tissue produced an average number of disconnected pores of $347 \pm 40$ for cortex tissue and $668 \pm 158$ for core tissue. These results showed statistical differences $(\mathrm{P}$-value $<0.05)$ between the analyzed regions, and also revealed the higher 
number of disconnected pores in the tissue close to the core, being 1.9 times more disconnected than those close to the peel.

The forms of the distributions in Fig. 7a-b are very significant for building accurate network models for studying fluid motion. To date, such models have largely been built on 6connected 3D lattices having constant lattice spacing (see, for example, the review article of Celia et al. (1995)). Both of these modeling assumptions are inconsistent with the results shown here.

\section{Conclusions}

X-ray computed microtomography has been used to quantitatively characterize the architecture of apple tissue, variety Jonagold, from two different regions of the sample: in the fleshy part closer to the peel $(\sim 6 \mathrm{~mm}$ from the peel) and near to the core $(\sim 10 \mathrm{~mm}$ from the core center) using a representative elementary volume of $1.3 \mathrm{~mm}^{3}$. This technique has shown to be an effective and efficient tool not only to nondestructively visualize the interior of the apple, but can also provides the micrometer resolution to quantitatively analyze and characterize the microstructure of the apple tissue. Also, the extracted parameters and spatial distributions are obtained directly from the data set without recourse to geometrical assumptions. The 3-D imaging of intercellular spaces clearly showed that the intercellular spaces were interconnected and formed a highly complicated network inside the tissues. Comparisons among the pore network parameters extracted from the tissue near to the peel and the tissue near to the core showed significant differences $(\mathrm{P}$-value $<0.05)$. These results quantitatively confirm the expectation of reduced void spaces as one proceeds from the tissue near to the peel toward the interior tissue. The porosity and specific surface area for cortex tissue and core tissue were $31 \pm 3 \%$ and $1.8 \times 10^{-2} \pm 1.2 \times 10^{-3} \mu^{-1}$ and $17 \pm 4 \%$ and $1.4 \times 10^{-2}$ $\pm 2.7 \times 10^{-3} \mu \mathrm{m}^{-1}$, respectively.

For the effective pore radius and tortuosity distributions associated with the cortex and core apple tissues, the gamma distribution, with its characteristic relatively steep rise and an exponentially falling tail provided a good characterization of the data. Relevant average parameters and fit distribution information are summarized in Table 1.

Finally, we conclude that the complexity and variability of the pore space network among these two tissue samples taken in different positions highlighted the difficult of creating a unique network for 3-D representation of apple tissue, and therefore, the physically-realistic pore network characterization of apple tissue cannot be adequately represented using simple average parameters of the total tissue.

\section{Acknowledgements}

The authors wish to thank to K.U. Leuven (Project OT 04/31) and the Fund for Scientific Research Flanders (F.W.O. - Vlaanderen, Project G.0200.02) for financial support for this investigation. Author Pieter Verboven is postdoctoral researcher of the Fund for Scientific Research Flanders (F.W.O. - Vlaanderen). 


\section{Literature cited}

Babin, P., Della Valle, G., Dendievel, R., Lassoued, N., \& Salvo, L. 2005. Mechanical properties of bread crumbs from tomography based finite element simulations. Journal of Materials Sciences, 40(22): 5867-5873.

Baoping, J. 1999. Nondestructive technology for fruits grading. In Proceedings of 99 International Conference on Agricultural Engineering, pp. IV 127- IV 133. Beijing, China, December.

Barcelon, E. G., Tojo, S., \& Watanabe, K., 1999. X-ray computed tomography for internal quality evaluation of peaches. Journal of Agricultural Engineering Research, 73(4): 323-330.

Celia, M., Reeves, P., \& Ferrand, L. 1995. Recent advances in pore scale models for multiphase flow in porous media. U.S. Natl. Rep. Int. Union Geod. Geophys. 1991-1994, Reviews of Geophysics, 33: 1049-1057.

Khan, A. A. \& Vincent, J. F. V. 1990. Anisotropy of apple parenchyma. Journal Science of Food Agriculture, 52: 455-466.

Kuroki, S., Oshita, S., Sotome, I., Kawagoe, Y., \& Seo, Y. 2004. Visualization of 3-D network of gas-filled intercellular spaces in cucumber fruit after harvest. Postharvest Biology and Technology, 33(3): 255-262.

Lammertyn, J., Dresselaers, T., Van Hecke, P., Jancsók, P., Wevers, M., \& Nicolaï, B. 2003. Analysis of the time course of core breakdown in 'Conference' pears by means of MRI and X-ray CT. Postharvest Biology and Technology, 29(1): 19-28.

Lee, T.-C., Kashyap, R., \& Chu, C.-N., 1994. Building skeleton models via 3-d medial surface/axis thinning algorithms. CVGIP: Graphic Models and Image Processing, 56(6): $462-478$.

Lim, K. S., and Barigou, M. 2004. X-ray micro-computed tomography of aerated cellular food products. Food Research International, 37: 1001-1012.

Lindquist, W. D. 1999. 3DMA General Users Manual. State of New York at Stony Brook: New York, pp. 1-47.

Lindquist, W. D., Lee, S.-M., Coker, D., Jones, K., and Spanne, P., 1996. Medial axis analysis of three dimensional tomographic images of drill core samples. Journal of Geophysical Research, 101B: 8297-8310.

Lorense, W. E. \& Cline, H. E. 1987. Marching cubes: a high resolution 3-D surface construction. ACM Computer Graphics (SIGGRAPH '87 Conference Proceedings), 21(4): 163-169.

Maire, E., Fazekas, A., Salvo, L., Dendievel, R., Youssef, S., Cloetens, P., and Letang, J.M. 2003. X-ray tomography applied to the characterization of cellular materials. Related finite element modeling problems. Composites Science and Technology, 63: 2431-2443.

Oh, W. \& Lindquist, W., 1999. Image thresholding by indicator kriging. IEEE Transactions on Pattern Analysis and Machine Intelligence, 21(7): 590-602.

Salvo, L., Cloetens, P., Maire, E., Zabler, S., Blandin, J. J., Buffière, J. Y., Ludwig, W., Boller, E., Bellet, D., \& Josserond, C. 2003. X-ray micro-tomography an attractive characterisation technique in material science. Nuclear Instruments and Methods in Physics Research, B 200: 273-286. 
Thovert, J., Salles, J., \& Adler, P., 1993. Computarized characterization of the geometry of real porous media: their discretization, analysis and interpretation. Journal of Microscopy, 170(1): 65-79.

van Dalen, G., Blonk, H., van Aalst, H., \& Hendriks, C. L. 2003. 3-D imaging of foods using X-ray microthomography. Imaging \& Microscopy, 3: 18-21.

Table 1. Resume of average values and parameters for fit distributions of microstructural features of apple tissue extracted from the cortex and core.

\begin{tabular}{|c|c|c|c|c|c|c|c|}
\hline & \multicolumn{3}{|c|}{ Cortex tissue } & \multicolumn{3}{|c|}{ Core tissue } & \multirow{2}{*}{$\begin{array}{c}\text { Test and } \\
\text { statistical } \\
\text { differences }\end{array}$} \\
\hline & Observations & $\begin{array}{l}\text { Distribution } \\
\text { type }\end{array}$ & Parameters & Observations & $\begin{array}{l}\text { Distribution } \\
\text { type }\end{array}$ & Parameters & \\
\hline Porosity & 12 & - & $\begin{array}{l}\mu=31 \% \\
s=3 \%\end{array}$ & 12 & - & $\begin{array}{l}\mu=17 \% \\
s=4 \%\end{array}$ & (a) \\
\hline $\begin{array}{l}\text { Specific surface } \\
\text { area }\left(\mu \mathrm{m}^{-1}\right)\end{array}$ & 12 & - & $\begin{array}{l}\mu=1.8 \times 10^{-2} \\
S=1.2 \times 10^{-3}\end{array}$ & 12 & - & $\begin{array}{l}\mu=1.4 \times 10^{-2} \\
s=2.7 \times 10^{-3}\end{array}$ & (a) \\
\hline $\begin{array}{l}\text { Nodal pore volume } \\
\left(\mathrm{mm}^{3}\right)\end{array}$ & 236 & Normal & $\begin{array}{l}\mu=-3.07 \\
s=0.91\end{array}$ & 194 & Normal & $\begin{array}{l}\mu=-3.4 \\
s=0.81\end{array}$ & (b) \\
\hline $\begin{array}{l}\text { Effective pore } \\
\text { radius }\end{array}$ & 412 & Gamma & $\begin{array}{c}a=3.35 \\
b=25.10\end{array}$ & 234 & Gamma & $\begin{array}{c}a=4.23 \\
b=16.09\end{array}$ & (b) \\
\hline $\begin{array}{l}\text { Tortuosity (shortest } \\
\text { paths in } \mathrm{x}-\mathrm{,y}-, \mathrm{z}- \\
\text { directions) }\end{array}$ & 224 & $\begin{array}{c}\text { Lognormal } \\
\text { Gamma }\end{array}$ & $\begin{array}{c}\mu=0.74 \\
s=0.19 \\
a=28.45 \\
b=0.08\end{array}$ & 182 & $\begin{array}{l}\text { Lognormal } \\
\text { Gamma }\end{array}$ & $\begin{array}{c}\mu=0.65 \\
s=0.16 \\
a=41.63 \\
b=0.05\end{array}$ & $\begin{array}{l}\text { (b) } \\
\text { (b) }\end{array}$ \\
\hline $\begin{array}{l}\text { Nodal pore } \\
\text { coordination } \\
\text { number }\end{array}$ & 226 & - & $\begin{array}{l}\mu=4 \\
s=2\end{array}$ & 346 & - & $\begin{array}{l}\mu=3 \\
s=2\end{array}$ & $(a, b)$ \\
\hline Path length $(\mu \mathrm{m})$ & 464 & - & $\begin{array}{l}\mu=18.77 \\
s=5.34\end{array}$ & 370 & - & $\begin{array}{c}\mu=12.18 \\
s=5.07\end{array}$ & (b) \\
\hline $\begin{array}{l}\text { Number of } \\
\text { disconnected pores }\end{array}$ & 12 & - & $\begin{array}{c}\mu=347 \\
s=40\end{array}$ & 12 & - & $\begin{array}{l}\mu=668 \\
s=158\end{array}$ & (a) \\
\hline
\end{tabular}

(a) Denote statistical differences using t-test (P-value of 0.05)

(b) Denote statistical differences using Kolmogorov-Smirnov test (P-value of 0.05) 